\title{
BMJ Open Family-based, healthy living intervention for children with overweight and obesity and their families: a 'real world' trial protocol using a randomised wait list control design
}

\begin{abstract}
Sam Liu, ${ }^{1}$ Isabela Gouveia Marques (D) , ${ }^{1}$ Megan A Perdew, ${ }^{1}$ Karen Strange, ${ }^{2}$ Teresa Hartrick, ${ }^{2}$ Joy Weismiller, ${ }^{3}$ Geoff D C Ball, ${ }^{4}$ Louise C Mâsse, ${ }^{5}$ Ryan Rhodes, ${ }^{1}$ Patti-Jean Naylor ${ }^{1}$
\end{abstract}

To cite: Liu S, Marques IG, Perdew MA, et al. Family-based healthy living intervention for children with overweight and obesity and their families: a 'real world' trial protocol using a randomised wait list control design. BMJ Open 2019;9:e027183. doi:10.1136/ bmjopen-2018-027183

- Prepublication history for this paper is available online. To view these files, please visit the journal online (http://dx.doi org/10.1136/bmjopen-2018027183).

Received 15 0ctober 2018 Revised 18 September 2019 Accepted 02 October 2019
Check for updates

(c) Author(s) (or their employer(s)) 2019. Re-use permitted under CC BY-NC. No commercial re-use. See rights and permissions. Published by BMJ.

For numbered affiliations see end of article.

Correspondence to Dr Patti-Jean Naylor; pjnaylor@uvic.ca

Dr Sam Liu; samliu@uvic.ca

\section{ABSTRACT}

Introduction Family-based behavioural weight management interventions are efficacious and widely used to address childhood obesity. Curriculum and strategies vary extensively and scale-up often depends on ensuring that the intervention fits the adoption context.

Aims and objectives To evaluate the impact and implementation of a 'made in British Columbia' (BC) family-based early intervention programme (EIP) for $8-12$ years old with overweight and obesity and their families.

Methods and analysis A randomised waitlist-control trial will assess a 10-week interactive, family-based lifestyle intervention followed by four maintenance sessions, in BC, Canada. We aim to enrol 186 families. The blended intervention includes at least 26 contact hours between participants and programme providers, including interactive activities and educational materials through weekly 90-min group sessions, an online family portal, and self-directed family activities. Curricular content includes information and activities related to healthy eating, physical activity (PA), positive mental health, parenting practices and sleep hygiene. The waitlist control group will receive a modified programme with the same 10-week sessions in the family portal, and four group sessions. Families participate in data collection at baseline, postintervention (week 10) and follow-up (week 18). The primary outcome is to assess changes in child body mass index $z$-score at 10 weeks between the groups. Secondary outcomes include changes at 10 weeks between the groups in child and parent PA behaviour and skills, healthy eating behaviour, and mental health. Process evaluation will address reach, implementation and maintenance (baseline, 10-week and 18-week) using recruitment tracking forms, parent questionnaire, programme attendance tracking forms, leader feedback surveys, parents and children satisfaction surveys and postprogramme interviews with facilitators, stakeholders and parents. Intention-to-treat analyses will be conducted. Process evaluation will be analysed thematically.

\section{Strengths and limitations of this study}

- The randomised wait-list control design is a strong and ethical design.

- Intervention informed by best available evidence and community stakeholders.

- Innovative components include positive mental health and blended in-person/online delivery.

- Participant enrolment and drop-out are challenges that can increase selection and attrition bias, respectively.

Ethics and dissemination Study procedures were designed to address research and community needs and will follow ethical standards.

Trial registration number NCT03643341.

\section{INTRODUCTION}

Obesity is one of the most common paediatric health problems ${ }^{1}$ and has been linked to multiple physiological and psychosocial problems throughout childhood. ${ }^{2}$ Over $25 \%$ of the children are either overweight or obese in British Columbia (BC), Canada. There is also a significant disparity in the prevalence of overweight and obesity across population groups (eg, Indigenous children and those in the lowest income bracket). ${ }^{3}{ }^{4}$ Without intervention, overweight children will likely continue to be overweight during adolescence and adulthood..$^{56}$

Family-based behavioural weight management interventions are a main approach for achieving weight control in children and adolescents. ${ }^{7}$ Encouraging the whole family to make behavioural changes decreases the 
focus being placed solely on children's dietary and activity behaviours ${ }^{8}$ and also focuses on providing a supportive environment for making lifestyle modifications in the home setting. Several randomised controlled trials have shown that family-focused behavioural programme delivered in-person can be effective strategies to manage childhood obesity. ${ }^{2}{ }^{9-12}$ Although these intervention programme can be effective in managing childhood obesity, the delivery methods must be scalable to enhance public health impact. ${ }^{13-15}$ Unfortunately, in-person familyfocused childhood weight management programme have limited reach (eg, only available at specific locations) and are resource intensive (eg, programme require significant human input). ${ }^{1415}$ Consequently, there is an urgent need to develop innovative solutions to improve the scalability of these childhood obesity management programme to enhance public health impact.

With the advancement in internet-enabled digital devices (eg, smartphones, tablets, computers, wearables) and improved access to the internet, there is emerging evidence these innovative digital technologies can help improve the scalability of in-person family-based childhood obesity management programme without overtaxing healthcare resources. ${ }^{13} 16$ There are currently two main methods of using the internet to deliver familybased health childhood obesity management interventions: (1) a stand-alone internet-based programme and (2) a blended intervention internet and face-to-face programme. ${ }^{17} 18$

Stand-alone internet-based interventions can be advantageous to administer over long distances, allow families to work at their own pace, save travelling time, and reduce the stigma of going to a childhood obesity management programme. However, families may feel a lack of support compared with face-to-face programme. ${ }^{17}$ Attrition with such programme is often a concern for stand-alone internet-based programme. ${ }^{19}$ By contrast, a blended faceto-face and internet-based programme can retain the positive aspects associated with both forms of therapy while mitigating the disadvantages. Adding internet interventions might improve adherence to behaviour change as internet, or mobile elements could be used to support behaviour change during face-to-face sessions and thereby increase the effectiveness of face-to-face intervention. ${ }^{17} 18$ Currently, there is inadequate data to determine the efficacy blended internet-based interventions aimed to manage childhood obesity by targeting the entire family. ${ }^{15}$ Thus, it is critical to evaluate these approaches and understand how these modes of delivery can complement each other in a 'real-world' setting.

The proposed research provides the opportunity to examine the efficacy of a blended (in-person and webbased), 'made in BC', family healthy living early intervention programme (EIP) in managing obesity (body mass index (BMI) $\geq 85$ th percentile for age and sex) in children 8-12 years of age. EIP was developed to enhance implementation using an extensive needs assessment and stakeholder engagement process with over 300 stakeholders across the province who provided input based on their current clinical and professional practice and experience. EIP was designed to (1) align with existing evidence and theory-based (multi-process action (M-PAC) framework) practices in the clinical and public health setting (eg, a minimum of 26 hours of contact time, family involvement, physical activity (PA), healthy living, sleep, mental health); (2) complement existing childhood obesity management programme in BC (HealthLink BC Eating and Activity Programme for Kids: telephone-based support programme for overweight children, shapedown: a community based designed for children with BMI $\geq 97$ th percentile for age and sex); (3) meet the needs of $\mathrm{BC}$ families and communities, by making the programme accessible to diverse families (eg, indigenous, multicultural or intercultural backgrounds, lower-income, single-parent); (4) address existing gaps documented in family-focused intervention literature (eg, address lifestyle without focusing on weight, incorporate extensive mental health and resilience-based activities for families, trauma-informed practice training for leaders, blended delivery models $)^{2021}$; (5) incorporate the latest internetbased features (eg, wearable data integration, interactive quizzes, reminders and notifications, online discussion forum). Stakeholder's input also emphasised the importance of: compatibility with existing resources, flexibility to adapt for different communities, a focus on healthy lifestyles rather than weight, one face-to-face contact per week to reduce family and community burden and enhance relative advantage.

The purpose of the proposed trial is to examine the efficacy of the experimental intervention versus wait-list control group on health and behaviour outcomes over a 10 -week period. The primary outcome is to asses changes in child BMI z-score. Secondary outcomes include changes in child fundamental movement skills (FMS); PA engagement, predilection, adequacy, intrinsic motivation, competence, confidence; sedentary habits and screen time, confidence, and family support; self-esteem, gratitude, self-compassion and sleep. Also changes in dietary behaviours, healthy eating outcome expectation, motivation, self-efficacy and perceived cooking skills will be assessed. Parent outcomes assessed include PA support, habit and identity; changes in parent feeding practices, structure of the home food environment, parents' personal dietary behaviours, food preparation self-efficacy, habit and identity. Our primary hypothesis is that children participating in the EIP will maintain or reduce their BMI z-score after 10 weeks, compared with those in the waitlist control group. Our secondary hypotheses are that EIP participants (parents and children) will make more positive lifestyle changes in PA and healthy eating, as well as parenting practices and mental health, after 10 weeks, relative to the waitlist participants. We also hypothesise that the EIP will reach a broad demographic, and families and staff will be satisfied with the EIP. 


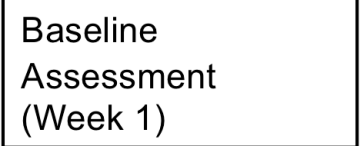

\section{Post-Intervention assessment (Week 10)}

Follow-up assessment (Week 18)

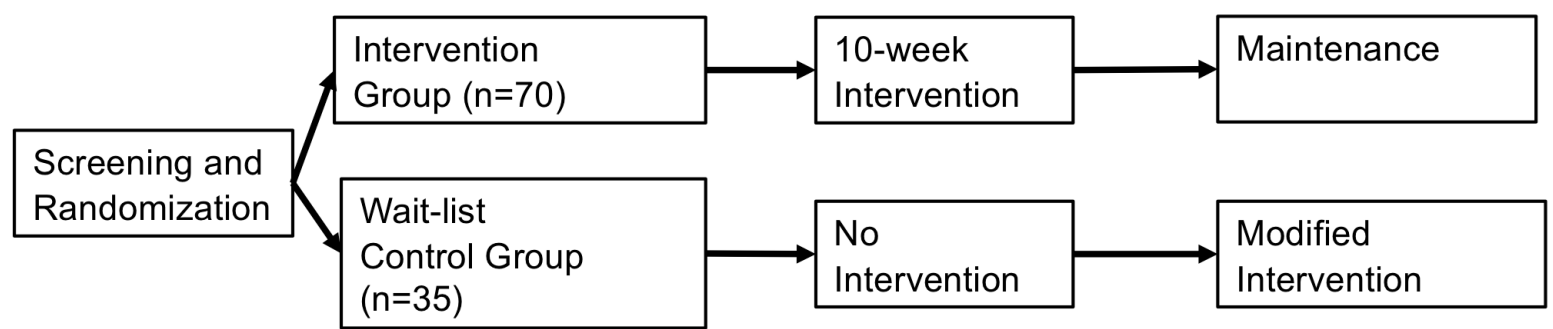

Figure 1 Overview of the early intervention programme study design.

\section{METHODS AND ANALYSIS}

The Standard Protocol Items: Recommendations for Interventional Trials (SPIRIT) reporting guidelines was used to report the study protocol. ${ }^{22}$

\section{Study design}

A randomised waitlist-controlled trial will assess the 10-week interactive family-based lifestyle intervention followed by four maintenance sessions (figure 1), in BC, Canada, from October 2018 to September 2020. The intervention includes at least 26 contact hours between participants and programme providers, including interactive activities and educational materials through weekly 90-min group sessions, an online family portal and selfdirected family activities.

The parameters used for sample size calculation was based on the results of a published randomised controlled trial evaluating the efficacy of a family-based intervention to reduce BMI z-score relative to control. ${ }^{2}$ Based on 2:1 randomisation, and anticipating $20 \%$ drop out, the estimated sample for the intervention group is $n=124$ and the waitlist control group is $\mathrm{n}=64$ (using a two-parallel group design, type 1 error $=5 \%$ and power $=80 \%$ ). Randomisation will be blocked (random permuted block design) within each of our six recruitment across BC representing all five health authority regions: Prince George (YMCA of Northern BC); Kelowna (YMCA of the Okanagan); Surrey (Tong Louie YMCA); Surrey (City of Surrey); Burnaby (City of Burnaby); Greater Victoria (Westshore Recreation and Parks Society) to ensure overall balance (2:1) in the number of participants assigned to the two groups. Randomisation will be conducted by an independent researcher. The randomisation code will be hidden from research assistants during assessments and data processing of the primary and secondary outcomes. In this study, an allocation of 2:1 in favour of the intervention group will be used because of the availability of resources and the minimal number of participants required to carry out an intervention at each site. Blinding families is not possible as intervention and waitlist programme start dates are different. Blinding the research team is also not possible due to real world constraints on scheduling whereby the measurement will be scheduled during scheduled group time and waitlisted families are scheduled at a further time. Thus, this is one of the study limitations. In order to minimise the chance of group contamination, participants will be instructed to not discuss details of their treatment with others outside the study. All participants' identifiers will be removed during data analyses.

\section{Inclusion/exclusion criteria}

Participants will be children aged $8-12$ years old, with a BMI $\geq 85$ th percentile for age and sex, ${ }^{23}$ accompanied by a parent, family member, or legal guardian. At least one member of the family will have to be able to speak and read English, and families will have to agree to attend group meetings over 10 weeks. Families will be excluded if medical clearance was needed and not obtained, and if the child has a BMI $<85$ th percentile.

\section{Waitlist control group}

An ethical imperative for any study of a family-based obesity EIP is to ensure that the control arm receives essential information about preventive guidelines for childhood obesity management. Thus, the waitlist control group will have access to a modified programme at week10: four group sessions and full access to the 10-week online family portal after the study is completed.

\section{Recruitment}

Participants will be recruited using: Active Living Guide inserts; school newsletter inserts; local newspaper advertisements and interviews; mailed packages to physician offices, community health centres, diabetes clinics, allied health professionals; letters and email blasts to Provincial networks and organisations; posters and rack cards displayed in recreation centres, public community spaces, medical offices and schools; a customised website; social media domains such as Facebook, Instagram and Twitter; webinars; booths at events and summer camps; and using local radio. Parents may contact the study team directly about enrolment via the study website, email or phone call. Also, parents who express interest will be asked to provide their name and contact details to the recreation 
centre staff and will receive a follow-up email or phone call delivering more information about programme eligibility and enrolment. Parents will be asked to confirm their participation in the programme within a week from completing the screening call. Next, parents will be asked to sign consent forms and children will sign the child assent form, confirming that they have discussed the intervention with their parents and understand the programme's requirements.

\section{Intervention: EIP}

The EIP design represents a community-based delivery model and was designed based on a systematic review of the literature, ${ }^{24}{ }^{25}$ based on findings from previous implementation efforts ${ }^{26} 27$ in BC and extensive community stakeholder consultations across five health regions (more than 300 stakeholders). The EIP development was guided theoretically by the M-PAC framework 2829 that emphasises social cognitive approaches to intention formation, adoption of action control through selfregulation and the action control maintenance phase once a behaviour becomes habitual and self-identified. Intervention activities were designed to support children and parents in learning behavioural change skills that will enable them to improve their health-related lifestyle behaviours. The M-PAC constructs are reflected in the EIP's curriculum to introduce and direct participants in making long-term lifestyle behaviour changes. The M-PAC establishes seven constructs that are antecedent of behaviours: (1) instrumental attitude as the knowledge on health consequences, (2) affective judgement relating to intrinsic motivation, (3) perceived capability relating to self-efficacy, (4) perceived opportunity relating to perceptions of the social and physical environment (time and access), (5) behavioural regulation relating to tactics that people use to translate their intentions into behaviour (eg, goal setting, self-monitoring), (6) identity as a standard of conscious self-comparison and (7) habit as a stimulusenacted behavioural response under lowered conscious awareness. A recent review of 23 studies that have applied M-PAC provided general support of its tenets and strong support for the multivariate associations between these antecedents and behaviour. ${ }^{30}$

Following the systematic review evidence, the 10-week intervention includes at least 26 contact hours ${ }^{31}$ between participants and intervention activities and materials through in-person and online activities. Group sessions will be held once a week for $90 \mathrm{~min}$ and they include family PA, children-only PA aiming at improving enjoyment, confidence, motivation and FMS, and parent-only group discussion to identify barriers and strategies for promoting family healthy behaviours. Additional hours will be obtained via the online family portal.

\section{Curriculum}

The intervention targets lifestyle changes in both children and their parents in regards to promoting healthy eating, reduction of sugary drink consumption, increasing cooking self-efficacy, engaging in family PA, reduction of recreational screen time and sedentary behaviour, improved sleep hygiene, positive mental health, self-esteem, gratitude and self-compassion. The weekly topics covered are listed in table 1. Behaviour change techniques used in the programme include goal setting, self-monitoring, self-evaluation, communication and interpersonal skills. The EIP will also provide four extra community-based group sessions. Two of these extra sessions will be a session in a local park using the Agents of Discovery mobile application, which is an augmented reality mobile application designed to encourage families to engage in outdoor exploration, and a group grocery store tour led by a registered dietitian. The remaining two group activities will be chosen and scheduled by the facilitators based on group input. Researchers designing the EIP intend to create a flexible community-based familyintervention programme able to accommodate families' demanding schedules.

\section{Online family portal}

The EIP online family portal will be considered as a weekly lesson to be completed by families. Lessons in the portal will offer additional resource information, healthy recipes, parent articles, videos, and suggested healthy eating and physical activities so that families engage in an extra 60 min per week of self-directed healthy lifestyle activities to promote healthy living. The online family portal will also be a repository of materials covered in each session, such as weekly handouts and worksheets. The portal will provide families with (1) a step tracking tool (eg, steps, active minutes, diet), (2) an interactive map of healthy places in their communities on, (3) online weekly quizzes to help families assess and strengthen their selfguided learning, (4) a secure online diary to allow families to reflect on their progress and set new weekly goals and (5) proactive online messages to notify families about new content, login and survey assessments.

\section{Maintenance sessions}

The intervention group will receive four 1 hour, biweekly maintenance sessions, after the 10-week programme. Sessions will include $30 \mathrm{~min}$ of discussion on maintaining healthy lifestyle, and 30 min of family PA.

\section{Data collection protocol}

Child and parent outcome measures will be collected at baseline, after the intervention (week 10). Process evaluation metrics such as family satisfaction, issues, facilitators and barriers to attendance and maintenance will be collected during and after the intervention (at 10 and 18 weeks). Parent questionnaires will be sent online prior to the intervention start. After screening for eligibility, both intervention and wait-list control group parents will receive an email containing instructions followed by a link for completing the online parent questionnaire.

Data from intervention and waitlist control children will be collected at the Healthy Living Workshop, 
Table 1 Weekly topics covered in the family-based early intervention programme

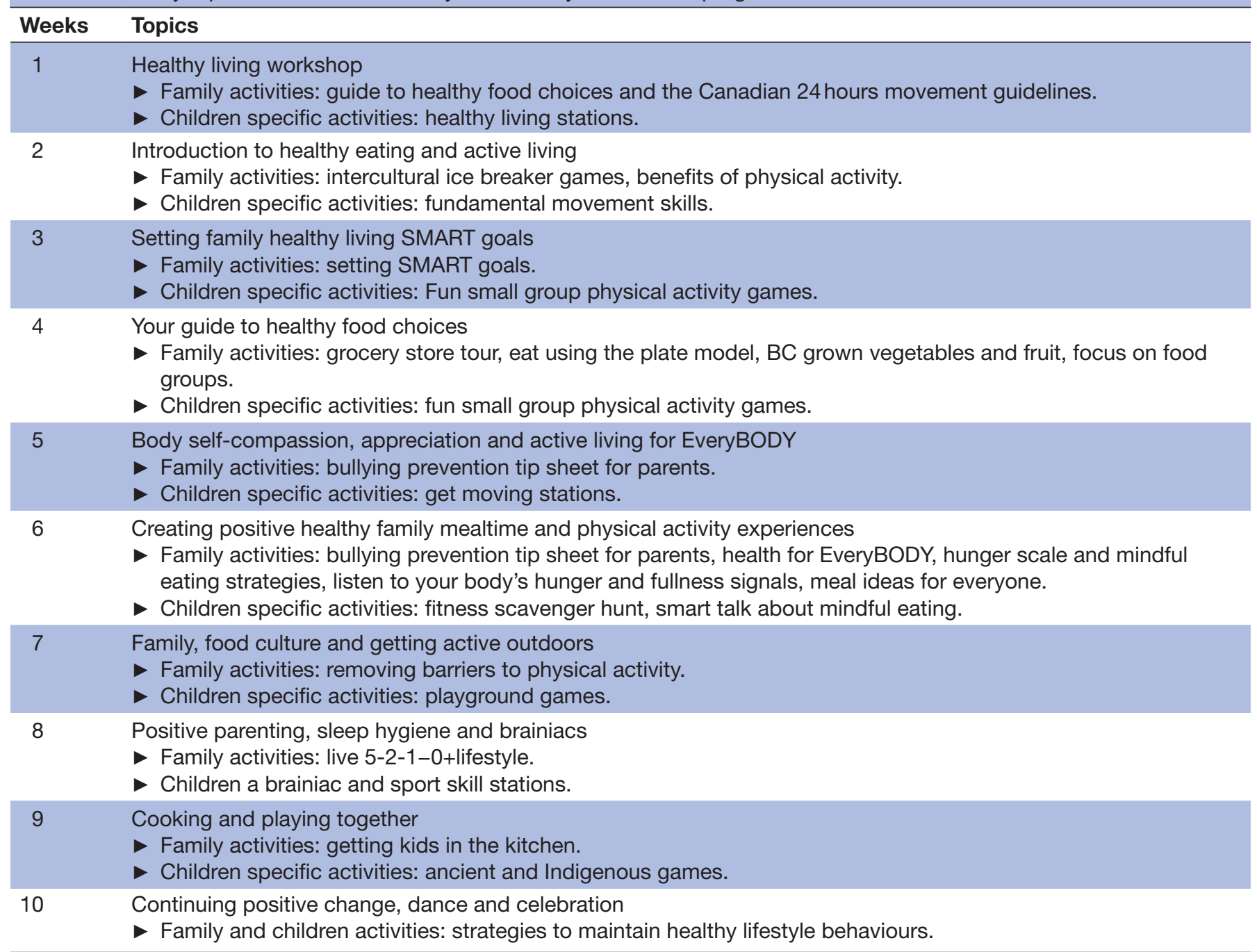

an interactive and fun 'health fair style' measurement approach that rotates between stations such as nutrition and PA games interspersed among questionnaire stations, FMS assessment, and BMI. All parents will be invited to attend a Healthy Living Workshop session while children participate in the health fair. The measurement team will follow-up with families who do not attend the measurement session. Programme facilitators will follow-up with families who do not come to the intervention. Data will be entered within 2 weeks of data collection. De-identified data will be securely stored at the University of Victoria server. Processes to promote data quality include double data entry; range checks for data values. Co-investigators will have access to de-identified final trial dataset.

\section{Outcome measures}

Child measures

- BMI will be calculated as weight (kilograms) divided by height (metres) squared, adjusted for child age and sex weight to the nearest $0.1 \mathrm{~kg}$ and height to the nearest $0.1 \mathrm{~cm}$ will be obtained. BMI z-scores (SD) will be calculated based on the Centers for Disease Control and Prevention criteria. ${ }^{23}$

- FMS will be assessed using the validated Canadian Agility and Movement Skill Assessment that evaluates seven skills: two-foot jumping, sliding, catch, throw, skip, one-foot hop and kick. ${ }^{32}$ Children will observe two demonstrations, will complete two practice trials, and two timed and scored trials.

- PA levels will be measured using the Physical Activity Questionnaire for Children. ${ }^{33}$

- Sedentary behaviours will be assessed using the Physicianbased Assessment \& Counseling for Exercise (PACE) Adolescent Psychosocial Measures. ${ }^{34}$

- Perceived PA intrinsic motivation and competence will be measured by the Motivation and Confidence subscale of the Canadian Assessment of Physical Literacy. ${ }^{32}$

- Dietary behaviours will be measured using the 7-day recall questionnaire retrieved from the Behavioral Risk Factor Surveillance System Survey Questionnaire. ${ }^{35}$

- Healthy eating outcome expectations and self-efficacy will be assessed using the Power Play! Survey, ${ }^{36}$ and 
the PACE Adolescent Psychosocial Measures, ${ }^{37}$ respectively.

- Healthy eating motivation will be assessed by the Family Life, Activity, Sun, Health, and Eating (FLASHE) questionnaire. $^{38} 39$

- Perceived cooking skills will be assessed by the Cooking with Kids questionnaire. ${ }^{40}$

- Quality of life will be assessed using the Pediatric Quality of Life Inventory. ${ }^{41}$

- Self-compassion, gratitude, self-esteem will be assessed using the Self-compassion Scale Short Form, ${ }^{42}$ the FLASHE questionnaire, ${ }^{38}$ subscales of the Project EAT survey, ${ }^{43}$ and the Gratitude Adjective Checklist. ${ }^{44}$

\section{Parent measures}

- Parent's PA and dietary behaviours will be assessed by subscales drawn from the FLASHES-EAT surveys ${ }^{45}$ and the Action Control of Parent Support Behaviour. ${ }^{46}$

- Structure of the home food environment will be assessed by the Fruit and Vegetable At Home Survey for Parents. ${ }^{47}$

- Parent PA and dietary support and behavioural regulation of supporting child's PA will be measured using the Parent Support of Child Physical Activity questionnaire. ${ }^{46} 48$

- PA and dietary habit will be assessed by the automaticity subscale of the Self-Report Index of Habit. ${ }^{49}$

- PA and dietary identity will be assessed by the RoleIdentity subscale from the Exercise Identity Scale. ${ }^{5051}$

\section{Process evaluation}

The EIP will be assessed using process evaluation components identified by Linnan and Steckler ${ }^{52}$; and components of the RE-AIM framework, ${ }^{53}$ specifically the reach, efficacy, implementation and maintenance components (see table 2).

Reach assesses the effectiveness of marketing strategies, the effectiveness of programme processes in generating appropriate referrals to the intervention, the extent that the intervention is reaching intended populations, and adherence and attrition rates. Reach will be assessed using site-specific recruitment plans, recruitment tracking forms, screening and phone calls tracking, demographic questionnaires, and programme attendance tracking forms. Programme coordinators for each community will record site-specific recruitment plans. Recruitment plans will outline and track all recruitment efforts undertaken at a local level. Centralised recruitment efforts will be tracked using a recruitment tracking form that will record all public inquiries including phone calls, emails and social media interactions. Information recorded will include name, community, contact information, date and form of contact, how they heard about the programme, any follow-up communication, and the outcome of the inquiry. The screening call tracking will record the individual's reasons for interest, ability to commit and eligibility. Demographic questionnaires will be completed by parents or caregivers to determine participants' cultural backgrounds, gender, age, and household make-up, income levels, education levels, and employment status. Programme attendance tracking forms will be completed by the programme facilitators throughout the duration of the programme. Attendance trackers will track weekly participant attendance, reasons for missed sessions, and participant drop-out.

Table 2 Summary of the process evaluation

\begin{tabular}{|c|c|c|}
\hline Component & Definition & Assessment \\
\hline Reach & $\begin{array}{l}\text { Effectiveness of marketing strategies, } \\
\text { recruitment, the extent that the intervention } \\
\text { is reaching intended populations, and } \\
\text { adherence and attrition rates. }\end{array}$ & $\begin{array}{l}\text { Site-specific recruitment plans, recruitment tracking } \\
\text { forms, screening and phone calls tracking, demographic } \\
\text { questionnaires, and programme attendance tracking forms. }\end{array}$ \\
\hline Efficacy & $\begin{array}{l}\text { The impact of the EIP intervention on } \\
\text { family's health and well-being outcomes. }\end{array}$ & $\begin{array}{l}\text { Child's measures: BMI z-score, FMS, PA levels, sedentary } \\
\text { behaviours, Intrinsic motivation and self-efficacy for PA and } \\
\text { dietary behaviours, quality of life, self-compassion, gratitude, } \\
\text { self-esteem. } \\
\text { Parent's measures: PA and dietary behaviours, structure of } \\
\text { the home food environment, parent support for the child's PA } \\
\text { and dietary behaviours, home food environment, habit and } \\
\text { identity for PA and dietary behaviours. }\end{array}$ \\
\hline
\end{tabular}

$\begin{array}{ll}\text { Implementation } & \begin{array}{l}\text { EIP satisfaction, programme fidelity, } \\ \text { attendance, barriers to programme } \\ \text { participation. }\end{array} \\ \text { Maintenance } & \begin{array}{l}\text { Conditions needed for successful long-term } \\ \text { implementation of the EIP. }\end{array}\end{array}$

Screening tracking form, facilitators preworkshop and postworkshop surveys, programme attendance tracking forms, facilitator feedback surveys, parents and children satisfaction surveys and postprogramme interviews with parents, facilitators and stakeholders.

- Maintenance will be assessed using stakeholders and advisory committee interviews.

BMI, body mass index; EIP, early intervention programme; FMS, fundamental movement skills; PA, physical activity. 
Implementation addresses if families, staff, and stakeholders are satisfied with the EIP, implementation fidelity, facilitators and barriers to participate in the programme, attendance, programme delivery team perceptions of parent benefits and satisfaction, and negative outcome tracking. Implementation will be assessed using screening tracking form, facilitators preworkshop and postworkshop surveys, programme attendance tracking forms, facilitator feedback surveys, parents and children satisfaction surveys and postprogramme interviews with parents, facilitators and stakeholders. The screening tracking form will identify potential facilitators and barriers to participate in the programme. Programme facilitators will complete a workshop survey before and after a 3-day training workshop that will assess facilitator's knowledge and confidence with implementing the programme curriculum and the effectiveness of the training workshop in these regards. Programme attendance tracking forms will record participant attendance and reasons for drop-out, including possible barriers to attendance and completion of the programme. Weekly facilitator feedback surveys will evaluate the successes and challenges of the weekly in-class sessions, as well as the facilitator's delivery of components of the session: PA, healthy eating, and positive mental health components. Parent and child satisfaction surveys will be completed at the end of the 10-week programme and will assess participant satisfaction with the programme curriculum and delivery. Parents will be asked to participate in postprogramme phone interviews in order to gain a deeper understanding of their perceptions and experiences with the EIP.

Programme coordinators and facilitators from each site will also be asked to take part in postprogramme interviews to explore their perceptions of the success and challenges of the programme delivery and the effectiveness of the facilitator training workshop for providing them with the knowledge and tools needed to deliver the content. Focus groups with the facilitation teams and programme coordinators will be completed in-person immediately following the last session of the EIP programme, or via phone call the week following the completion of the programme. Provincial stakeholder interviews will be held in person or by phone and will be scheduled at the earliest available date following the completion of the programme, and will be conducted by the EIP project coordinator.

Maintenance evaluates the conditions needed for successful long-term implementation of the EIP by assessing stakeholder support and integration and alignment with BC's Continuum for the Prevention, Management, and Treatment of Health Issues Related to Overweight and Obesity in Children and Youth. ${ }^{54}$ Maintenance will be assessed using stakeholders and advisory committee interviews. Stakeholder and advisory committee interviews will be conducted by the EIP project coordinator. Interviews will be held in person or by phone and will be scheduled at the earliest available date following the completion of the programme.

\section{Patient and public involvement}

The EIP was designed based on previous childhood obesity weight management in BC and accounted for participants' feedback. Community stakeholders were actively involved in the study design. The EIP was prepiloted in the Spring 2018 and participants' feedback on recruitment, burden of the intervention and measurement were taking into consideration for the full trial.

\section{Data analysis}

We will analyse our outcomes using an intention-to-treat approach. We will use descriptive to evaluate our primary and secondary outcomes at baseline. We will evaluate patterns of missing data in the treatment groups and we will perform multiple imputation to address missing data if data are missing at random. The distributions of the continuous variables will be evaluated and we will apply a suitable transformation if the distribution is significantly skewed. For our primary outcome (BMI z-score), the difference among groups at 10 weeks will be evaluated using a univariate linear regression adjusted for baseline outcome measures (eg, BMI z-score at baseline), socialeconomic status and recruitment sites. Secondary outcomes (FMS, PA levels, perceived PA intrinsic motivation and competence, dietary, healthy eating motivation, perceived cooking, quality of life self-compassion, gratitude, self-esteem, parent's PA and dietary behaviours and behavioural regulation of supporting child's PA, PA and dietary habit) will follow a similar statistical approach as the primary outcome analysis.

Statistical significance criterion of will defined as $\mathrm{p}<0.05$. Process evaluation data will be described using descriptive statistics and thematic analysis will be done by two independent coders to identify, analyse and report themes. ${ }^{55}$ Coders will read the transcripts, identify possible themes, draft and compare the codebook, discuss potential themes, and draft the first official version of the codebook. Then, coders will code all the transcripts, discuss and develop version two of the codebook. A third researcher will be consulted if agreements cannot be reached. Finally, we will evaluate programme adherence as part of the process evaluation. We will be conducting a 'per protocol' analysis including only intervention participant to evaluate adherence (number of in-class and online sessions completed) during intervention and maintenance period.

\section{ETHICS AND DISSEMINATION}

All participants will provide electronic and written consent. Children will provide written assent. Ethics was obtained from the University of Victoria Ethics Review Board prior to participant recruitment. Amendments to the protocol will be submitted to the University of Victoria Ethics Review Board.

International recommendations agree that the core elements of any intervention to address childhood obesity should involve the whole family and include nutrition 
education, behaviour modification, and promotion of PA. Recent randomised controlled trials found familybased behavioural programme that targeted families with obese 8-12years old showed positive outcomes in both short-term (10weeks) and long-term (12 months) interventions. ${ }^{24}$

The Province of British Columbia Ministry of Health has provided funding to the Childhood Obesity Foundation to design and implement a 'made in BC' communitybased Childhood Healthy Weights Early Intervention Programme for children 8-12 years old. The EIP was developed following essential processes for scalability ${ }^{56}$ : it was based on the current family-based childhood obesity management literature, ${ }^{24}{ }^{25}$ based on lessons learnt from previous programme conducted in the province, ${ }^{26}$ it was overseen by a stakeholder Steering Advisory Committee and based on an extensive regional stakeholder consultation and needs assessment process. The programme will also include innovative topics on sleep hygiene and screen use as a holistic way to promote healthy lifestyles as well as a novel blended (internet-based and in-person) delivery approach. The EIP was designed using a new meta-theoretical (M-PAC). ${ }^{28}$

We anticipate that findings from the trial will have high impact, given our collaboration with the Childhood Obesity Foundation and the structure of the initiative and its development. Additionally, while the intervention is running there will be a sustainability subcommittee that is addressing systems of programme integration and client triage. Advancements achieved with this study, concerning the content and methodology of family-based obesity programme, if effective and feasible will likely be widely disseminated in $\mathrm{BC}$ dependent on ongoing funding.

\section{Author affiliations \\ ${ }^{1}$ School of Exercise Science, Physical and Health Education, University of Victoria, Victoria, British Columbia, Canada \\ ${ }^{2}$ Childhood Obesity Foundation, Vancouver, British Columbia, Canada \\ ${ }^{3}$ Juniper Consulting, Victoria, British Columbia, Canada \\ ${ }^{4}$ Department of Pediatrics, University of Alberta, Edmonton, Alberta, Canada \\ ${ }^{5}$ Faculty of Medicine, University of British Columbia, Vancouver, British Columbia, Canada}

Acknowledgements Steering committee: BC Ministry of Health, Childhood Obesity Foundation, University of Victoria, Juniper Consulting, ShapeDownBC, SCOPE 5210 , HealthLINK BC, YMCA of Greater Vancouver, BC Recreation and Parks Association (BCRPA). Research advisory committee: University of Victoria, University of Alberta, University of British Columbia. Management committee: Childhood Obesity Foundation, University of Victoria, Juniper Consulting. Oversight Committee: BC Ministry of Health, Provincial Health Services Authority (PHSA), Childhood Obesity Foundation, University of Victoria. We acknowledge participants in the EIP pre-pilot program that was conducted in North Cowichan in the Spring 2018, and who provided great feedback. GDCB was supported by an Alberta Health Services Chair in Obesity Research. LCM received salary support from the BC Children's Hospital Research Institute. RR was supported by a Kennedy $Y$ H Wong Distinguished Visiting Professorship from Hong Kong Baptist University during the writing of this paper and is also supported by funds from the Canadian Institutes for Health Research, the Canadian Cancer Society, the Social Sciences and Humanities Research Council of Canada and the Heart and Stroke Foundation of Canada.
Contributors P-JN and KS conceived the study. P-JN, KS, SL, JW, GDCB, RR, TH and LCM contributed to the study design. SL, P-JN, IGM, MAP drafted and revised the manuscript. All authors edited and approved the final manuscript.

Funding This trial is supported by funding from the British Columbia Ministry of Health through the Childhood Obesity Foundation. This funding source has been involved in the design of this study, and will have no role during its execution, analyses, interpretation of the data, or decision to submit results.

Competing interests P-JN is on the Board of Childhood Obesity Foundation and had course release to oversee the implementation of the evaluation of the EIP. P-JN reports grants from Childhood Obesity Foundation, during the conduct of the study. KS, GM, TS, JW and MAP report personal fees from Childhood Obesity Foundation, during the conduct of the study.

Patient consent for publication Not required.

Provenance and peer review Not commissioned; externally peer reviewed.

Open access This is an open access article distributed in accordance with the Creative Commons Attribution Non Commercial (CC BY-NC 4.0) license, which permits others to distribute, remix, adapt, build upon this work non-commercially, and license their derivative works on different terms, provided the original work is properly cited, appropriate credit is given, any changes made indicated, and the use is non-commercial. See: http://creativecommons.org/licenses/by-nc/4.0/.

\section{ORCID iD}

Isabela Gouveia Marques http://orcid.org/0000-0002-4174-0705

\section{REFERENCES}

1 Hughes AR, Stewart L, Chapple J, et al. Randomized, controlled trial of a best-practice individualized behavioral program for treatment of childhood overweight: Scottish childhood overweight treatment trial (Scott). Pediatrics 2008;121:e539-46.

2 Sacher PM, Kolotourou M, Chadwick PM, et al. Randomized controlled trial of the mend program: a family-based community intervention for childhood obesity. Obesity 2010;18 Suppl 1:S62-8.

3 Katzmarzyk PT. Obesity and physical activity among Aboriginal Canadians. Obesity 2008;16:184-90.

4 Ruben AR. Undernutrition and obesity in Indigenous children: epidemiology, prevention, and treatment. Pediatr Clin North Am 2009;56:1285-302.

5 Twig G, Yaniv G, Levine $\mathrm{H}$, et al. Body-Mass index in 2.3 million adolescents and cardiovascular death in adulthood. $N$ Engl $\mathrm{J}$ Med 2016;374:2430-40.

6 Canning PM, Courage ML, Frizzell LM. Prevalence of overweight and obesity in a provincial population of Canadian preschool children. Can Med Assoc J 2004;171:240-2.

7 Kalarchian MA, Levine MD, Arslanian SA, Silva A, et al. Family-Based treatment of severe pediatric obesity: randomized, controlled trial. Pediatrics 2009;124:1060-8.

8 Sung-Chan P, Sung YW, Zhao X, et al. Family-Based models for childhood-obesity intervention: a systematic review of randomized controlled trials. Obes Rev 2013;14:265-78.

9 Kalarchian MA, Levine MD, Arslanian SA, et al. Family-Based treatment of severe pediatric obesity: randomized, controlled trial. Pediatrics 2009;124:1060-8.

10 Croker H, Viner RM, Nicholls D, et al. Family-Based behavioural treatment of childhood obesity in a UK National health service setting: randomized controlled trial. Int J Obes 2012;36:16-26.

11 Savoye M, Shaw M, Dziura J, et al. Effects of a weight management program on body composition and metabolic parameters in overweight children: a randomized controlled trial. JAMA 2007;297:2697-704.

12 Golley RK, Magarey AM, Baur LA, et al. Twelve-month effectiveness of a parent-led, family-focused weight-management program for prepubertal children: a randomized, controlled trial. Pediatrics 2007;119:517-25.

13 Hohman $\mathrm{KH}$, Price SN, Sonneville K, et al. Can the Internet be used to reach parents for family-based childhood obesity interventions? Clin Pediatr 2012;51:314-20.

14 Verrotti A, Penta L, Zenzeri L, et al. Childhood obesity: prevention and strategies of intervention. A systematic review of schoolbased interventions in primary schools. J Endocrinol Invest 2014;37:1155-64.

15 Chen J-L, Wilkosz ME. Efficacy of technology-based interventions for obesity prevention in adolescents: a systematic review. Adolesc Health Med Ther 2014;5:159. 
16 Nolan RP, Liu S, Shoemaker JK, et al. Therapeutic benefit of Internet-based lifestyle counselling for hypertension. Can J Cardiol 2012;28:390-6.

17 Erbe D, Eichert H-C, Riper $\mathrm{H}$, et al. Blending face-to-face and Internet-based interventions for the treatment of mental disorders in adults: systematic review. J Med Internet Res 2017;19:e306.

18 Andersson G, Titov N. Advantages and limitations of Internet-based interventions for common mental disorders. World Psychiatry 2014;13:4-11.

19 Liu S, Dunford SD, Leung YW, et al. Reducing blood pressure with Internet-based interventions: a meta-analysis. Can J Cardiol 2013;29:613-21.

20 Flynn MAT, McNeil DA, Maloff B, et al. Reducing obesity and related chronic disease risk in children and youth: a synthesis of evidence with 'best practice' recommendations. Obes Rev 2006;7 Suppl 1:7-66.

21 Sacks G, Shill J, Snowdon W, et al. Prioritizing areas for action in the field of population-based prevention of childhood obesity, 2012.

22 Chan A-W, Tetzlaff JM, Altman DG, et al. Spirit 2013 statement: defining standard protocol items for clinical trials. Ann Intern Med 2013;158:200-7.

23 Kuczmarski RJ, Ogden CL, Guo SS, et al. 2000 CDC growth charts for the United States: methods and development. Vital Heal Stat 11 2002;246:1-190.

24 Janicke DM, Steele RG, Gayes LA, et al. Systematic review and meta-analysis of comprehensive behavioral family lifestyle interventions addressing pediatric obesity. J Pediatr Psychol 2014;39:809-25.

25 Ash T, Agaronov A, Young Ta'Loria, Young TL, et al. Family-Based childhood obesity prevention interventions: a systematic review and quantitative content analysis. Int J Behav Nutr Phys Act 2017;14.

26 Childhood Obesity Foundation. Mend scale up and implementation 2014-2016 evaluation report, 2017.

27 Childhood Obesity Foundation. Childhood healthy weights intervention initiative: shifting the destination by shifting the trajectory evaluation report, 2015.

28 Rhodes RE. The evolving understanding of physical activity behavior, 2017.

29 Rhodes RE, de Bruijn G-J. What predicts intention-behavior discordance? A review of the action control framework. Exerc Sport Sci Rev 2013:41:201-7.

30 Rhodes RE. Bridging the intention-behavior gap in physical activity: a review of evidence from the multi-process action control framework. Appl Physiol Nutr Metab 2018;39:105-7.

31 Wilfley D, Balantekin KN. Family-Based behavioral interventions for childhood obesity. In: Pediatric obesity. Humana press, Cham, 2018: 555-67.

32 Healthy Active Living and Obesity Research Group. Canadian assessment of physical literacy. Ottawa: Manual for Test Administration, 2017. www.haloresearch.ca

33 Kowalski KC, Crocker PRE, Donen RM, et al. The physical activity questionnaire for older children (PAQ-C) and adolescents (PAQ-A) manual. Coll Kinesiol Univ Saskatchewan 2004;87:1-38.

34 Norman GJ, Vaughn AA, Roesch SC, et al. Development of decisional balance and self-efficacy measures for adolescent sedentary behaviors. Psychol Health 2004;19:561-75.

35 Centers for Disease Control and Prevention (CDC). Behavioral risk factor surveillance system survey questionnaire, 2011.

36 Keihner AJ, Meigs R, Sugerman S, et al. The Power Play! Campaign's School Idea \& Resource Kits improve determinants of fruit and vegetable intake and physical activity among fourth- and fifth-grade children. J Nutr Educ Behav 2011;43:S122-9.
37 Hagler AS, Norman GJ, Radick LR, et al. Comparability and reliability of paper- and computer-based measures of psychosocial constructs for adolescent fruit and vegetable and dietary fat intake. J Am Diet Assoc 2005; 105:1758-64.

38 Mâsse LC, Lytle LA. Advancing knowledge of parent-child Dyadic relationships about multiple cancer preventive health behaviors: the National cancer Institute family life, activity, sun, health, and eating (FLASHE) study. Am J Prev Med 2017;52:833-5.

39 Wilson AM, Magarey AM, Mastersson N. Reliability and relative validity of a child nutrition questionnaire to simultaneously assess dietary patterns associated with positive energy balance and food behaviours, attitudes, knowledge and environments associated with healthy eating. Int J Behav Nutr Phys Act 2008;5:5-12.

40 Lohse B, Cunningham-Sabo L, Walters LM, et al. Valid and reliable measures of cognitive behaviors toward fruits and vegetables for children aged 9 to 11 years. J Nutr Educ Behav 2011;43:42-9.

41 Varni JW, Limbers CA, Burwinkle TM. Parent proxy-report of their children's health-related quality of life: An analysis of 13,878 parents' reliability and validity across age subgroups using the PedsQL??? 4.0 Generic Core Scales. Health Qual Life Outcomes 2007;5.

42 Froh JJ, Fan J, Emmons RA, et al. Measuring gratitude in youth: assessing the psychometric properties of adult gratitude scales in children and adolescents. Psychol Assess 2011;23:311-24.

43 Pasch Ket al. Adolescent sleep, risk behaviors, and depressive symptoms: are they linked? Am J Health Behav 2010;34:237-48.

44 Sutton E, Schonert-Reichl KA, AD W, et al. Evaluating the reliability and validity of the Self-Compassion scale short form adapted for children ages 8-12. Child Indic Res 2017.

45 Nebeling LC, Hennessy E, Oh AY, et al. The FLASHE study: survey development, Dyadic perspectives, and participant characteristics. Am J Prev Med 2017;52:839-48.

46 Rhodes RE, Spence JC, Berry T, et al. Understanding action control of parental support behavior for child physical activity. Health Psychology 2016;35:131-40.

47 Robinson-O'Brien R, Neumark-Sztainer D, Hannan PJ, et al. Fruits and vegetables at home: child and parent perceptions. J Nutr Educ Behav 2009;41:360-4.

48 Rhodes RE, Blanchard CM, Matheson DH. A multicomponent model of the theory of planned behaviour. Br J Health Psychol 2006;11:119-37.

49 Gardner B, Abraham C, Lally P, et al. Towards parsimony in habit measurement: testing the convergent and predictive validity of an automaticity subscale of the self-report habit index. Int J Behav Nutr Phys Act 2012;9.

50 Anderson DF, Cychosz CM. Development of an exercise identity scale. Percept Mot Skills 1994;78:747-51.

51 Wilson P, Muon S. Exercise identity measurement: psychometric properties of the exercise identity scale in a university sample. Int $J$ Sport Exerc Psychol 2008;6:115-31.

52 Linnan L, Steckler A. Process evaluation for public health interventions and research. San Francisco: Jossey-Bass, 2002

53 Glasgow RE, Vogt TM, Boles SM, et al. Evaluating the public health impact of health promotion interventions: the RE-AIM framework, 1999. Available: www.or

54 Bradbury J, Day M, Scarr J. British Columbia's Continuum for the Prevention, Management. and Treatment of Health Issues Related to Overweight and Obesity in Children and Youth 2015 www. childhealthbc.ca

55 Braun V, Clarke V. Using thematic analysis in psychology using thematic analysis in psychology 2006;0887:77-101.

56 Milat AJ, Li B. Narrative review of frameworks for translating research evidence into policy and practice. Public Heal Res Pract 2017;27:1-13. 